\title{
Maintenance with Rituximab in anti-neutrophil cytoplasm antibody-associated vasculitis
}

\author{
Maria Skopis ${ }^{1}$, Ayse Bag-Ozbek ${ }^{2}$ \\ 'Department of Internal Medicine, Mount Sinai Medical Center, Miami Beach, FL 33140, USA. \\ 2Department of Internal Medicine, Division of Rheumatology, Allergy and Immunology, SUNY Stony Brook University Hospital, \\ Stony Brook, NY 11794, USA.
}

Correspondence to: Dr. Ayse Bag Ozbek, Department of Internal Medicine, Division of Rheumatology, Allergy and Immunology, SUNY Stony Brook University Hospital, 101 Nicolls Road, Stony Brook, NY 11794, USA. E-mail: abagozbek@gmail.com How to cite this article: Skopis M, Bag-Ozbek A. Maintenance with Rituximab in anti-neutrophil cytoplasm antibody-associated
vasculitis. Vesse/ Plus 2020;4:30. http://dx.doi.org/10.20517/2574-1209.2020.42.

Received: 20 Aug 2020 First Decision: 9 Sep 2020 Revised: 21 Sep 2020 Accepted: 23 Sep 2020 Published: 16 Oct 2020

Academic Editor: Luca Quartuccio Copy Editor: Cai-Hong Wang Production Editor: Jing Yu

\begin{abstract}
Granulomatosis with polyangiitis, microscopic polyangiitis and renal-limited anti-neutrophil cytoplasm antibody (ANCA) associated vasculitis are the main ANCA-associated vasculitidies (AAV). Multiple induction therapies for AAV exist and have proven successful in achieving disease remission. Azathioprine and methotrexate have been used to maintain remission of $A A V$, however, relapse rates and adverse effects with these medications remain high. Rituximab (RTX), a B cell depleting monoclonal antibody, was shown to be safe and effective in maintaining disease remission in AAV in early retrospective reviews. In 2014, the first randomized control trial to compare RTX and azathioprine in maintenance therapy of newly diagnosed AAV (MAINRITSAN trial), revealed that patients who received RTX after cyclophosphamide induction had higher rates of sustained remission, fewer adverse effects and, better overall survival rates as compared to azathioprine. MAINRITSAN 2 revealed that patients receiving tailored regimens of maintenance RTX received fewer infusions but did not have higher rates of relapse than patients who received fixed dose therapy. The RITAZAREM trial conveyed that patients who experienced $A A V$ relapse after induction therapy that received induction and maintenance RTX were significantly less likely to develop a relapse at 24 months $v s$. patients who received maintenance therapy with azathioprine. Overall, these studies suggest that maintenance therapy with RTX represents an exceptional treatment option in patients with $A A V$ in terms of safety and efficacy, resulting in lower relapse rates and less drug toxicity than conventional treatments. As a result, patients have fewer exposures to cytotoxic medications and thus, improved outcomes.
\end{abstract}

Keywords: Rituximab, ANCA vasculitis, maintenance therapy, B cell depletion, azathioprine, methotrexate

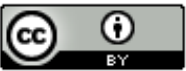

(C) The Author(s) 2020. Open Access This article is licensed under a Creative Commons Attribution 4.0 International License (https://creativecommons.org/licenses/by/4.0/), which permits unrestricted use, sharing, adaptation, distribution and reproduction in any medium or format, for any purpose, even commercially, as long as you give appropriate credit to the original author(s) and the source, provide a link to the Creative Commons license, and indicate if changes were made.

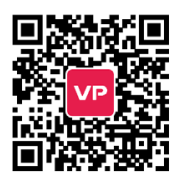




\section{INTRODUCTION}

Anti-neutrophil cytoplasmic antibody associated (ANCA) vasculitis is characterized by infiltration of neutrophils into small blood vessel walls, resulting in autoinflammation and necrosis ${ }^{[1]}$. Microscopic polyangiitis (MPA), granulomatosis with polyangiitis (GPA), eosinophilic granulomatosis with polyangiitis (EGPA), and renal-limited ANCA-associated vasculitis are considered the main ANCA associated vasculitides (AAV). Granulomatosis with polyangiitis and MPA present similarly and have significant disease overlap, therefore, they are often considered together in terms of management. Eosinophilic GPA is a process defined by eosinophilic pneumonia and its manifestations are dictated by eosinophilic inflammation; treatment strategies for this disease are therefore focused on this distinct aspect of its pathogenesis and differ from the treatment of the other ANCA associated vasculitides.

These rare diseases mostly affect individuals over the age of 50 and can progress to life threatening, fulminant multisystem disease with complications such as diffuse alveolar hemorrhage (DAH) and necrotizing glomerulonephritis causing respiratory failure and renal failure, leading to significant mortality rates $^{[2]}$. Furthermore, despite significant treatment advances in remission induction, preventing disease relapse and successfully maintaining disease remission continues to provide a significant challenge to physicians. Anti-neutrophil cytoplasmic antibody associated vasculitides therefore must be viewed as chronic diseases with complex treatment strategies that require long term immunosuppression.

Given the rarity of these diseases and the complexity of their treatment, significant efforts have been made over the years to investigate safe, effective induction and maintenance therapies for these patients. Prior to these significant advancements, AAV ran a fulminant and fatal course with rapidly progressive multisystem disease and an extremely high mortality rate. Eighty-two percent of patients with untreated GPA would die in 1 year and $90 \%$ would die in 2 years ${ }^{[3]}$. Initially, induction therapy with glucocorticoids was attempted and improved survival rates to 1 year, however, this strategy was associated with a multitude of side effects and a high rate of disease relapse ${ }^{[4]}$.

In the early 1970s and 1980s, promising evidence of cyclophosphamide (CYC) use in conjunction with glucocorticoids for induction therapy surfaced. A paramount study in 1985 revealed that $93 \%$ of patients with GPA followed over a 21-year period who received induction therapy with CYC and glucocorticoids achieved disease remission ${ }^{[3]}$. Unfortunately, however, the cytotoxic side effects of long-term CYC use became apparent including leukopenia, hemorrhagic cystitis, infertility and 31-fold increased risk of bladder cancer ${ }^{[4]}$. Despite being an effective agent in inducing disease remission in AAV, the cytotoxicity of CYC provided long term harm to patients and thus, was not an ideal agent for maintaining disease remission. Further research was needed to discover new treatment modalities that would deliver less harmful side effects.

Multiple studies explored alternate induction therapies for AAV, most notably the CYCLOPS trial in 2009 (comparing IV pulse doses of CYC vs. daily oral CYC and prednisolone as induction therapy) ${ }^{[5]}$, the MORAM trial (exploring efficacy of methotrexate (MTX) in inducing remission vs. CYC and prednisolone) ${ }^{[6]}$, and the MEPEX and PEXIVAS trials which studied the use of plasma exchange $v s$. glucocorticoid therapy for patients with AAV and sought to determine whether plasma exchange resulted in any significant difference in renal recovery, progression to ESRD or, overall incidence of death in patients with ANCA associated vasculitis ${ }^{[7,8]}$. Although promising, these alternate therapies did not prove to be any more efficacious than CYC while still having high relapse rates and significant drug toxicities.

Two randomized controlled trials exploring induction therapy with RTX vs. CYC (RAVE and RITUXVAS trials) found that induction therapy with RTX (administered alone in the RAVE trial and co-administered with CYC in the RITUXVAS trial) was non-inferior to CYC alone in inducing disease remission and that 
RTX did not have an increased risk of adverse events as compared to CYC. Additionally, at month 18 in the RAVE trial and at month 12 in the RITUXVAS trial, RTX alone was shown to be as efficacious in preventing disease relapse as CYC followed by azathioprine (AZA) maintenance therapy ${ }^{[9,10]}$. The RAVE trial also found that RTX had a better safety profile than $\mathrm{CYC}^{[9]}$. This was a cornerstone discovery in that, an induction therapy as effective as CYC (especially in severe relapsing disease) had yet to be discovered with the added benefit that RTX had a better safety profile. Despite these promising discoveries for induction therapy, maintenance of AAV remission still poses a significant challenge to physicians. This has prompted further research into alternative maintenance therapies.

\section{Remission maintenance therapies in ANCA associated vasculitis}

Cyclophosphamide, an alkylating agent, has been used as the gold standard of induction therapy in AAV for many years. Given its significant toxicity, however, it is not an ideal agent to use in maintaining disease remission in AAV, as long-term exposure significantly increases the risk of adverse effects including hemorrhagic cystitis and bladder cancer. Therefore, research of alternate remission maintenance strategies has been aimed at maintaining disease remission with less toxic immunosuppressants after initial induction therapy with cyclophosphamide.

In 2003, a randomized trial comparing AZA versus CYC in maintaining disease remission in ANCAassociated vasculitis was conducted. The study included patients who had recently been diagnosed with generalized vasculitis that were also found to have renal involvement (with a serum creatinine of $5.7 \mathrm{mg} / \mathrm{dL}$ or less). All patients received CYC and glucocorticoids for induction of disease remission. After achieving remission, patients were randomly assigned to receive CYC $(1.5 \mathrm{mg} / \mathrm{kg})$ or AZA $(2 \mathrm{mg} / \mathrm{kg} / \mathrm{day})$ maintenance therapy while both groups continued receiving prednisolone. These patients were followed for 18 months. Relapse rates in both groups were roughly the same (11 patients in the AZA group and 10 patients in the CYC group). The study thus concluded that administering AZA after induction therapy with CYC was effective in maintaining disease remission and that AZA could be used as a feasible and less toxic maintenance therapy than CYC for patients with ANCA-associated vasculitis ${ }^{[1]]}$.

Methotrexate has also been researched for use as a maintenance therapy in AAV. In 2017, a single center, open-label randomized trial studied the use of MTX $v s$. CYC for maintenance therapy in AAV. The study enrolled patients with MPA, GPA or EGPA and administered induction therapy with CYC before randomizing these patients to receive maintenance therapy with CYC or MTX for 12 months. The patients were monitored for relapses for 12 months after receiving induction therapy. The study found that the frequency of relapses and adverse events was the same between two groups. Methotrexate was thus designated a safe and effective alternate option for maintenance therapy in AAV after CYC induction ${ }^{[12]}$.

Further studies sought to investigate the use of AZA vs. MTX in maintenance of disease remission in ANCA-associated vasculitis. The Wegner Granulomatosis-Entretien (WEGENT) trial, an open label, multicenter study, investigated patients with a diagnosis of MPA or GPA who had been given induction therapy with CYC and glucocorticoids and were then randomized 1:1 to receive maintenance AZA ( $2 \mathrm{mg} / \mathrm{kg} /$ day) or MTX ( $0.3 \mathrm{mg} / \mathrm{kg} /$ day increased incrementally to $25 \mathrm{mg} /$ week) for $12 \mathrm{months}$. The patients were followed for 29 plus or minus 13 months and monitored for relapses. The results showed that similar relapse rates and numbers of adverse events occurred in both the AZA and MTX groups, indicating that the two drugs have similar efficacies in terms of maintenance of disease remission and, similar safety profiles in terms of adverse events ${ }^{[13]}$.

The above data demonstrates that while AZA and MTX are alternate options for maintenance therapy in patients with AAV after CYC induction and, that they do decrease the overall amount of exposure to CYC, they are still associated with a high risk of relapse and adverse events, with neither of these medications 
proving to be safer or more efficacious than the other. This fact prompted further investigation into safer, more efficient remission maintenance therapies such as RTX.

\section{RITUXIMAB IN ANCA ASSOCIATED VASCULITIS}

Rituximab is a monoclonal antibody consisting of both human and murine components that specifically targets the B cell CD20 antigen, thus causing removal of B cells from the peripheral circulation ${ }^{[14]}$. This is important because B cells have been implicated in the pathogenesis of AAV via their production of ANCAs, activation of the alternative complement pathway, and initiation of inflammatory cascades that induce severe necrotizing vascular inflammation. The ANCA autoimmune response is driven by inhibited suppression of $\mathrm{T}$ cells and $\mathrm{B}$ cells, and by release of B-cell stimulating factors by activated neutrophils which act to slow the apoptosis of $B$ cells, thus enhancing their proliferation ${ }^{[15]}$. Due to the fact that the pathogenesis of AAV is rooted in B cell mediated autoimmunity, it stands to reason that RTX would be of significant therapeutic benefit given its ability to target autoreactive B cells and deplete them from the circulation. As such, RTX provides a promising treatment option for induction and maintenance therapy in AAV because it targets the direct pathogenesis of the disease.

\section{MAINTENANCE THERAPY WITH RITUXIMAB IN AAV}

With the advent of promising research demonstrating RTX as a safe and effective induction therapy, investigators set their sights on this drug as a possible maintenance therapy for patients with AAV. Rituximab showed promise as a maintenance therapy for AAV in early case reports and retrospective reviews. In 2001, the use of RTX was described in a man with a history of chronic, relapsing GPA who did not tolerate treatment of disease relapse with CYC due to bone marrow toxicity, which caused significant anemia. Additional immunosuppressive treatments such as AZA and mycophenolate mofetil (MMF) were attempted, however, the patient did not respond to these treatments and had continued relapses with worsening renal failure and a meningeal flare of GPA. The decision was made to initiate therapy with RTX on a compassionate basis given the patient had been refractory to all conventional treatments and MTX was contraindicated. Subsequently, the patient received 4 infusions of $375 \mathrm{mg} / \mathrm{m}^{2}$ of RTX and high dose glucocorticoids. The patient entered complete remission after treatment with RTX and it was observed that his cytoplasmic ANCA (cANCA) and B lymphocyte levels had completely disappeared. At 11 months after treatment, the patient's cANCA and B lymphocytes reappeared, yet he did not have any signs or symptoms of disease relapse; he was preemptively treated with RTX at that time and remained in complete remission at 18 -month follow up ${ }^{[16]}$ [Table 1].

In 2005, a case series reviewed 9 patients with a history of GPA or MPA who had either been resistant to treatment with CYC or, had experienced recurrent relapses after cessation of CYC therapy. Two of the patients were myeloperoxidase (MPO) - ANCA positive and 7 were proteinase-3 (PR-3) ANCA positive. These patients were treated with RTX infusions in addition to conventional immunosuppressants (AZA, MMF, or a short course of CYC) in order to prevent the formation of antibodies to RTX. Three patients were treated twice with a once per week infusion and four patients were treated four times with a once per week infusion. Eight out of the 9 patients in the study exhibited a complete response to RTX therapy with one patient having a partial response. Responses included improvement of chest x-ray (four patients), cessation of lower extremity gangrene (one patient), improvement of peripheral neuropathy (one patient), remission of renal vasculitis (two patients), and improvement in severe musculoskeletal pain (one patient) ${ }^{[17]}$ [Table 1].

A retrospective study of 8 patients with a history of relapsing or refractory GPA who received RTX infusions in conjunction with ongoing immunosuppressive therapy was conducted in 2007 . This study investigated the efficacy of RTX in patients with GPA who had either failed treatment with prior 
Table 1. Studies regarding maintenance therapy with Rituximab in ANCA-associated vasculitis

\begin{tabular}{l}
\hline Study and year of study \\
\hline Response of Wegner's \\
granulomatosis to \\
Anti-CD20 chimeric \\
monoclonal antibody \\
therapy, $2001^{[16]}$
\end{tabular}

Nine patients with antineutrophil cytoplasmic antibody-associated vasculitis successfully treated with rituximab, $2005^{[17]}$

Adjunction of rituximab to steroids and immunosuppressants for refractory/relapsing Wegner's granulomatosis: a study on 8 patients, $2007^{[18]}$
A multicenter survey of Rituximab therapy for refractory antineutrophi cytoplasmic antibodyassociated vasculitis, $2009^{[19]}$
Rituximab as maintenance therapy for antineutrophil cytoplasmic antibody-associated vasculitis, $2010^{[20]}$

Rituximab for remission induction and maintenance in refractory granulomatosis with polyangiitis (Wegner's), 2012: ten year experience at a single center ${ }^{[21]}$ with rituximab therapy
from January 1, 2000 to May 31, 2010 39 patients received maintenance therapy with rituximab

Single-center historical cohort study observing all patients (53 total) relapsing GPA treated
Objective of study

use of rituximab to

treat a patient with chronic, relapsing GPA

who did not tolerate cyclophosphamide therapy and was resistant to treatment with glucocorticoids, azathioprine and mycophenolate mofetil

To review the outcomes of 9 patients with MPA and GPA treated with rituximab who were either resistant to or had recurrent relapses after cessation of cyclophosphamide To investigate rituximab use in conjunction with ongoing steroid and immunosuppressant therapy as a treatment for relapsing/refractory GPA and to determine the frequency of infusions, time to patient response and, effects on the various manifestations of GPA
Rituximab was able to successfully induce and maintain disease remission in a patient with chronic, relapsing GPA resistant to other immunosuppressants
Study limitations

The report only describes the response of one individual to rituximab which fails to generalize the results to other patients with ANCA associated vasculitis
Case series which included with structured patient follow up

Retrospective study of 8 patients with refractory or relapsing GPA who received rituximab infusions in addition to their ongoing immunosuppressive therapy
Standardized collection from 65 patients option in treating patien at 4 centers in the UK with with ANCA associated a history of refractory AAV vasculitis

who received rituximab as induction therapy (largest series reported at that time) retrospective data

Rituximab was efficient and safe as an induction and maintenance therapy for patients with MPA and GPA

Treatment of relapsing/ refractory GPA with rituximab in conjunction with steroids/ immunosuppressants resulted in good clinical outcomes

There was a dissociation in the time to response of vasculitis manifestations (improved over days to weeks) vs. granulomatous manifestations (improved over several months) to rituximab

Rituximab was found to be successful as an induction therapy in patients with AAV Additionally, patients who received preemptive retreatment in the absence of any signs of a relapse with a regimen of $1 \mathrm{~g}$ rituximab every 6 months had no disease relapse at eleven- month follow up, suggesting rituximab as a viable maintenance therapy

To determine the efficacy with a history of chronic

\section{and safety of rituximab} infusions as maintenance therapy in patients with ANCA-associated vasculitis who had achieved complete or partial remission

To determine the efficacy of rituximab as a therapy for maintenance of remission in patients with a history of chronic relapsing refractory GPA
Rituximab was safe and effective in maintaining disease remission in patients with ANCAassociated vasculitis

Rituximab was effective and well tolerated as an induction and maintenance therapy in patients with a history of chronic relapsing GPA
No control arm

Possible selection bias Patients received additional immunosuppressive medications while they were on treatment with rituximab

Patients were receiving concomitant immunosuppressive therapy so it is difficult to tell if results could be attributed to rituximab alone

Possibility of positive outcome bias given that the study was a retrospective review

Comparison with other studies is limited because this cohort had lower disease activity at study onset

Open-label administration of rituximab Experience of study was only from one center with a predominantly Caucasian population of Scandinavian and Northern European background Except for 1 patient, all patients were PR-3 ANCA positive 


\section{Rituximab} maintenance therapy for granulomatosis with polyangiitis and microscopic polyangiitis, $2012^{[22]}$

MAINRITSAN trial, $2014^{[23]}$

MAINRITSAN2 trial, $2018^{[24]}$
Retrospective study of the To investigate the outcomes and tolerance efficacy of rituximab of patients with MPA and GPA treated with rituximab maintenance therapy

Nonblinded, randomized controlled trial

Open-label, pragmatic, multicenter randomized controlled trial as a maintenance therapy in patients with AAV who achieved disease remission with conventional immunosuppressants or rituximab

To compare rituximab infusions to azathioprine as maintenance therapy in patients with ANCA associated vasculitis (MPA, GPA, and renalassociated ANCA vasculitis)

To compare rituximab infusions tailored to the appearance of ANCA autoantibodies, increasing ANCA titers and/or the presence of CD19+ B cells in the circulation measured every three months to fixed dose regimens of rituximab in patients with GPA or MPA as maintenance therapy
RITAZAREM trial, 2019 ${ }^{[25]}$ International, multi-center, To compare the efficacy open-labeled, randomized, of rituximab $v s$. oral controlled trial azathioprine as a maintenance therapy in patients with a history of relapsing AAV who had received induction therapy with rituximab

Prolonged B cell depletion with rituximab is effective in treating refractory pulmonary granulomatous inflammation in granulomatosis with polyangiitis (GPA), $2014^{[26]}$

Rituximab for treatment of severe renal disease in ANCA associated vasculitis, 2016 ${ }^{[28]}$
Rituximab in the treatment of refractory scleritis in patients with polyangiitis (Wegener's), $2015^{[29]}$
Retrospective case series To investigate the efficacy of rituximab infusions in treating pulmonary granulomas in patients with GPA who were previously resistant to traditional immunosuppressive treatment

Retrospective multi-center To investigate the study efficacy of rituximab and glucocorticoids alone $v s$. rituximab, glucocorticoids and cyclophosphamide as a treatment for AAV patients with severe renal disease

Retrospective analysis of To evaluate the efficacy interventional case series of rituximab in patients with a history of GPA who developed scleritis that was refractory to conventional immunosuppressant therapy
Rituximab was well

Half of the patients tolerated and maintained had received additional remission of patients with immunosuppression GPA and MPA however, it concomitantly with did not completely prevent rituximab, making it relapses

${ }^{\star \star}$ The preliminary results of this study were to be confirmed by the MAINRITSAN trial which was in progress when the results of this study were published

Rituximab infusions of 500 mg given every 6 months were superior to azathioprine as maintenance therapy in AAV, particularly in patients who are PR-3 ANCA positive

There was not a significant difference in the number of relapses in the tailored infusion group $v s$. the fixed dose regimen group; tailored infusion group received fewer infusions while still maintaining a low relapse rate 
Rituximab therapy for refractory orbital inflammation: results of phase $1 / 2$, dose-ranging, randomized controlled trial, 2014 ${ }^{[30]}$

Successful treatment of hypertrophic pachymeningitis in refractory Wegener's granulomatosis with rituximab, $2009^{[31]}$

Effectiveness of Rituximab Retrospective analysis for the otolaryngologic manifestations of granulomatosis with polyangiitis (Wegener's) ${ }^{[32]}$

Diffuse alveolar hemorrhage secondary to antineutrophil cytoplasmic antibodyassociated vasculitis: predictors of respiratory failure and clinical outcomes, 2016 ${ }^{[33]}$

clinical trial

Single center historical cohort study
Dose ranging, randomized, To determine the efficacy Rituximab was double masked phase $1 / 2$ of rituximab in treating effective in treating orbital inflammation orbital inflammation due to GPA that was refractory to previous immunosuppressive treatment

To describe the use of The patient experienced rituximab to treat a patient complete remission after with GPA who developed treatment with rituximab pachymeningitis which was refractory to treatment with cyclophosphamide and pulsed dose methylprednisolone To determine the efficacy of rituximab in treating the ENT manifestations of for ENT manifestations of GPA

To determine the efficacy of plasma exchange, cyclophosphamide and rituximab in treating diffuse alveolar hemorrhage in patients with AAV
Small patient cohort (10 patients total with orbital inflammation, 2 of these patients had GPA)

Case report details the response of one individual patient to treatment thus these results cannot be extrapolated to other patients with this condition without further studies

Comments only on the ENT (granulomatous) manifestations of GPA, does not explore efficacy of rituximab for treatment of GPA manifestations secondary to systemic vasculitis

Study included predominantly patients with GPA rather than MPA

GPA: granulomatosis with polyangiitis; ANCA: anti neutrophil cytoplasmic antibody; MPA: microscopic polyangiitis; AAV: ANCA associated vasculitis; PR-3: proteinase-3; MPO: myeloperoxidase; MAINRITSAN: maintenance of remission using Rituximab in systemic ANCA-associated vasculitis; RITAZAREM: Rituximab vs. Azathioprine as therapy for maintenance of remission of anti-Neutrophil cytoplasm antibody-associated vasculitis

immunosuppressive therapy or, had continued to relapse despite treatment. The study found that RTX infusions improved the clinical outcomes of patients with relapsing or refractory GPA when used in conjunction with other immunosuppressants. Additionally, it was found that the granulomatous manifestations of GPA took longer to respond to RTX therapy (several months) as opposed to the vasculitis manifestations of GPA, which responded within weeks to months ${ }^{[18]}$ [Table 1].

A retrospective data collection two years later in 2009 (the largest data review to be conducted at that time) gathered data from 65 patients across 4 centers in the UK with a history of refractory AAV who received induction therapy with RTX. The study aimed to investigate the efficacy and safety of RTX in patients with refractory AAV as an induction therapy. Complete remission occurred in 49/65 patients (75\%), with partial remission occurring in $15 / 65$ patients (23\%). Only one patient did not respond to therapy. Furthermore, patients who received preemptive retreatment with $1 \mathrm{~g}$ of RTX every 6 months despite not having any symptoms of disease relapse had zero relapses at 11-month follow up, suggesting that RTX was also an effective maintenance therapy in patients with refractory $\mathrm{AAV}^{[19]}$ [Table 1].

A year later in 2010, another retrospective review studied RTX as a maintenance therapy in 39 patients with AAV who had already achieved either complete or partial remission. This study aimed to address the direct role of continuous infusions of RTX as a maintenance therapy in AAV patients. All 39 patients followed up after 1 year and 20 patients followed up after 2 years. The results showed that RTX treatment resulted in good disease control throughout the study. Median disease activity was measured according to the Birmingham Vasculitis Activity Score (BVAS), a comprehensive scoring system of all organ systems possibly affected by vasculitis that contains 59 items divided into 9 groups (i.e., general, cutaneous, mucous, 
renal, etc.). A lower score indicates lower disease activity, versus a higher score which indicates higher disease activity. Median disease activity of the patients in the study was 1 at baseline and improved to 0 at 12 and 24-month follow up. Eighty-seven percent of patients were on cytotoxic immunosuppression at the start of the study vs. $41 \%$ at 12 -month follow up and $30 \%$ at 24 -month follow-up ${ }^{[20]}$ [Table 1].

A single center historical cohort study in 2012 observed 53 patients with chronic relapsing GPA over 10-year period from January 1, 2000 to May 31, 2010 in whom RTX was used to maintain remission or, to treat disease relapses. The patients in the study received at least 2 courses of RTX (median number of courses of RTX was 4), with 52 of these patients being PR-3 ANCA positive. All patients who were treated for relapses achieved disease remission. Of the 53 patients, all achieved B cell depletion after induction therapy with RTX. Thirty two out of the 53 patients relapsed and, in all cases, relapses were associated with a rise in PR-3 ANCA levels and the reappearance of CD19+ B lymphocytes in the circulation. Remission was maintained successfully in all patients who were treated with preemptive courses of RTX based on re appearance of B lymphocytes and increases in PR-3 ANCA titers. Overall, this study conveyed the effectiveness of RTX as both an induction and maintenance strategy in patients with $\mathrm{AAV}^{[21]}$ [Table 1].

A retrospective study in 2012 examined the outcomes of 28 patients with AAV (4 with MPA and 24 with GPA) treated with RTX for maintenance therapy from 2003-2010. All patients in the study had entered remission with the use of conventional immunosuppressant therapy or RTX and were monitored for relapse rates and tolerance after greater than or equal to 2 RTX infusions used as maintenance therapy. The median range of RTX infusions was 4 with a median follow up time of 38 months from diagnosis or last flare. Out of 28 patients, 2 had pulmonary relapses, 1 patient suffered alveolar hemorrhage 6 months after a RTX infusion and 1 patient developed new lung nodules 11 months after a RTX infusion (this patient was then started on a new induction regimen with RTX which achieved disease remission and was followed by successful RTX maintenance). At final evaluation, 6 patients were in complete remission, 11 patients were in complete remission with irreversible damage and, 9 patients were in partial remission. 7 of these patients had persistent ENT involvement and 2 patients had persistent lung nodules. None of these patients had infusion reactions, 15 patients had hypogammaglobulinemia and 3 patients developed infections. The data of this study suggest that RTX could be used as a safe, effective maintenance therapy in $\mathrm{AAV}^{[22]}$ [Table 1]. Since the safety and efficacy of RTX as a maintenance therapy had been established in this study, the next question posed was the efficacy and safety of RTX as compared to other conventional maintenance therapies like AZA. This laid the framework for the largest randomized controlled trial to compare RTX to AZA as maintenance therapy in AAV titled the Maintenance of Remission using Rituximab in Systemic ANCA-associated Vasculitis (MAINRITSAN) trial.

In 2014, the MAINRITSAN trial investigated the use of RTX vs. AZA as a maintenance therapy in patients with AAV. The study was a non-blinded, randomized controlled trial that studied patients with GPA, MPA or renal-limited AAV who had received induction therapy with CYC and glucocorticoids and achieved disease remission. These patients were randomized to receive RTX infusions vs. daily AZA for maintenance therapy and were monitored for disease relapse. After 28 months, 5\% of the patients in the RTX group had suffered disease relapses vs. $29 \%$ of the patients in the AZA group. Additionally, adverse event rates were similar between the two groups. This study demonstrated that patients in the RTX group had significantly less relapses and an equal number of adverse events as the AZA group, thus conveying the superiority of RTX to AZA in maintaining disease remission in AAV while maintaining a similar safety profile to $\mathrm{AZA}^{[23]}$ [Table 1]. One interesting point is that the study was comprised mostly of patients who were anti PR-3 ANCA positive; patients who were anti MPO-ANCA positive and patients who had renal associated AAV comprised a smaller patient population in the study. An interesting point of further investigation would be to do additional trials exploring RTX as remission maintenance in patients who have anti MPO ANCApositive vasculitis to further determine their responsiveness to RTX treatment. 
Optimum regimen of rituximab maintenance therapy and reliability of ANCA titers and presence of CD19+ B lymphocytes as predictors of AAV relapse

In light of the evidence that RTX was superior to AZA in remission maintenance in the MAINRITSAN trial, investigators then set out to describe the optimum RTX treatment regimen in order to maintain disease remission in $\mathrm{AAV}$, and to determine whether the reappearance of ANCA autoantibodies, an increase in ANCA titers from baseline or, the reappearance of CD19+ B lymphocytes at follow up could provide a reliable marker of the need for reinfusion of RTX in order to prevent disease relapses.

The study was conducted by comparing the relapse rates of patients receiving individually tailored regimens of RTX infusions which were administered based on the reappearance of CD19+ B lymphocytes, reappearance of ANCA autoantibodies, or a marked increase in ANCA titers from baseline to the relapse rates of patients receiving fixed dose regimens of RTX. This trial was titled Maintenance in Remission using Rituximab in Systemic ANCA-associated Vasculitis-2 (MAINRITSAN2). The study was an open label, pragmatic, multicenter, randomized controlled trial. Patients were randomized in a 1:1 ratio to receive tailored RTX infusions or fixed dose regimens. These therapies were given 1 month after achieving remission with either CYC, MTX, or RTX induction in both groups.

The results of the study showed that $17.3 \%$ of the patients in the tailored therapy arm suffered relapse $v s$. 9.9\% of patients in the fixed dose regimen arm. Major relapses occurred in $7.4 \%$ of the tailored regimen arm vs. 3.7\% of the fixed dose regimen arm. Patients in the tailored infusion arm received 248 infusions $v s$. patients in the fixed dose regimen arm who received 381 infusions. The results of the MAINRITSAN2 trial demonstrated that individually tailored infusions of RTX based on biomarker activity were associated with low major relapse and relapse rates which did not differ significantly from the fixed dose regimen group, and allowed for administration of fewer RTX infusions. The study also demonstrated that reappearance of ANCA autoantibodies, increasing ANCA titers or, reappearance of CD19+B lymphocytes was not reliable in predicting AAV relapse, particularly because 4 patients in the study had negative ANCA autoantibodies and no circulating B cells at the time of disease relapse ${ }^{[24]}[$ Table 1].

\section{Rituximab maintenance therapy in patients with relapsing AAV}

In 2019, further studies were conducted to determine the efficacy of RTX vs. AZA as a maintenance therapy for patients with a history of relapsing AAV who achieved disease remission with RTX induction. This trial, known as the Rituximab as Therapy to Induce Remission after Relapse in ANCA-associated Vasculitis (RITAZAREM) trial, was an international, multi-center, open labeled, randomized controlled trial. Patients with a history of relapsing AAV were recruited for the study during the time of an active relapse and received glucocorticoids and RTX as induction therapy. The patients were then randomized to receive RTX or AZA maintenance in a 1:1 ratio and followed for 36 months, with $61 \%$ of patients having suffered a major relapse at the time of enrollment in the study. At 24 months after treatment, $13 \%$ of patients in the RTX group experienced relapses; of the 13\% of relapses in the RTX group, $82 \%$ were classified as minor and $18 \%$ were classified as major. $38 \%$ of patients in the AZA group suffered relapses; of the relapses in the AZA group, $62 \%$ were classified as minor and $38 \%$ were classified as major. The adverse event rate in the RTX group was lower (22\%) vs. the AZA group (36\%). The RITAZAREM trial successfully demonstrated that RTX maintenance therapy was superior to AZA in patients with a history of relapsing AAV who achieved remission with RTX induction and, that RTX was associated with fewer adverse events ${ }^{[25]}$ [Table 1].

\section{The role of rituximab in maintenance therapy of lung, renal, and other systemic manifestations of AAV}

Anti-neutrophil antibody associated vasculitis is a systemic disease which affects multiple organ systems. Below are studies outlining RTX as maintenance treatment of the various systemic manifestations of the disease. 
The role of RTX as a maintenance therapy of pulmonary granulomas in patients with GPA was described in an observational cohort study in 2014. The study looked at 5 patients with a history of PR-3 ANCA positive GPA who had pulmonary granulomas that were previously resistant to traditional immunosuppressive treatments. These patients received RTX infusions in reduced dosing schedules and were monitored for radiographic improvement of their pulmonary granulomas on chest x-ray (CXR) every 6 months for a total of 18-38 months. The results revealed that prolonged B cell depletion following treatment with RTX was effective in reducing both the size and number of pulmonary nodules in these patients for at least 18 months after treatment ${ }^{[26]}$.

Renal manifestations of AAV include necrotizing and rapidly progressive glomerulonephritis with crescent formation, leading to rapid decompensation of renal function and acute renal failure ${ }^{[2]}$. Renal involvement in AAV is an extremely poor prognostic factor, and it is the most significant predictor of mortality in AAV patients $^{[27]}$. The renal manifestations of AAV can occur without concomitant systemic vasculitis, an entity known as renal limited AAV. Rituximab has been studied as an induction agent for patients with AAV and severe renal disease. 37 patients with a history of AAV and a GFR $<20 / \mathrm{mL} / \mathrm{min} / 1.73 \mathrm{~m}^{2}$ were studied in order to determine the safety and efficacy of RTX and glucocorticoids alone $v s$. RTX, glucocorticoids and CYC as induction therapy in patients with AAV and severe renal disease. The study found that there was no difference in outcomes between the two treatment groups, and that RTX and glucocorticoids alone were as effective as RTX, CYC and glucocorticoids in treating AAV patients with severe renal disease ${ }^{[28]}$. The MAINRITSAN trial also included patients with renal associated AAV and displayed the superiority of RTX to AZA in maintenance of disease remission in these patients ${ }^{[23]}$.

Other manifestations of AAV successfully treated with RTX include refractory scleritis ${ }^{[29]}$, orbital GPA ${ }^{[30]}$, hypertrophic pachymeningitis in refractory $\mathrm{GPA}^{[31]}$, ENT manifestations of $\mathrm{GPA}^{[32]}$, and $\mathrm{DAH}^{[33]}$.

\section{ADVERSE EFFECTS OF RITUXIMAB}

Hypogammaglobulinemia has been observed in patients receiving maintenance therapy with RTX, which is of particular concern given the increased risk of developing serious infections when immunoglobulins are low $^{[2,24]}$. Literature detailing an ideal threshold immunoglobulin level at which to stop RTX in order to prevent development of serious, life threatening infections has not been described. It has been described that antibiotic prophylaxis is effective in preventing infections in patients who develop hypogammaglobulinemia after RTX therapy. These patients should receive influenza and pneumococcal vaccinations prior to the initiation of treatment in order to prevent infections $s^{[34]}$.

Late onset neutropenia has been described in patients with rheumatic diseases who have received RTX therapy, albeit this is a rare side effect. Several mechanisms have been proposed which could potentially contribute to late onset neutropenia in patients with rheumatic diseases receiving RTX including increased production of B lymphocytes which halts production of $\mathrm{T}$ cells and, infiltration of bone marrow and peripheral blood by T-large granular lymphocytes ${ }^{[34,35]}$.

Progressive multifocal leukoencephalopathy (PML) has also been described in patients with GPA and MPA receiving RTX treatment. A cumulative analysis conducted in 2018 which studied patients receiving RTX for GPA or MPA between 2009 and 2015 showed that the confirmed number of cases of reported PML was very low $(<1$ case per 10,000), indicating that this is a rather rare adverse effect of the medication. Despite its rarity, physicians should still be aware of the risk of development of PML in AAV patients receiving treatment with RTX, as these patients have often previously received cytotoxic immunosuppressants which are also known risk factors for development of $\mathrm{PML}^{[36]}$. 
Continued use of RTX increases the risk of serious infections depending upon the indication for its use and, the dose at which it is being administered. With regard to AAV patients, a retrospective case review demonstrated that severe infections occurred most commonly within the first year of receiving RTX treatment. Old age and lack of ENT involvement was a risk factor for developing severe infection ${ }^{[37]}$. Another study found that bronchiectasis and endobronchial involvement were notable risk factors for development of severe respiratory infections, and that antibiotic prophylaxis with Trimethoprimsulfamethoxazole was effective in preventing infections in patients with AAV who were receiving RTX infusions ${ }^{[38]}$.

\section{Rituximab as induction and maintenance therapy in EGPA}

Due to the fact that the pathogenesis of EGPA is dictated by eosinophil mediated inflammation and eosinophilic pneumonia as opposed to the other ANCA associated vasculitides (which are driven by the pathogenesis of $\mathrm{B}$ cell autoimmunity, neutrophil abnormalities and complement activation), the treatment of this specific subset of AAV can differ, which is why EGPA was excluded from most of the studies mentioned above. Induction therapy of life-threatening organ involvement in EGPA is commonly accomplished with CYC and glucocorticoids. A study in 2017 sought to investigate RTX vs. CYC as induction therapy in patients with EGPA who were refractory to prior induction therapy with CYC. This retrospective analysis studied 28 patients with EGPA and measured their treatment response when treated with RTX induction therapy vs. cyclophosphamide. Five of the patients in the RTX arm (36\%) achieved disease remission as opposed to four patients in the CYC arm (29\%). The remainder of the patients achieved partial remission. There was no difference in response to treatment between the two groups. Rituximab was well tolerated but did result in a decrease in serum immunoglobulin levels ${ }^{[39]}$. Further research is yet to be done on effective maintenance therapies for EGPA and would be an interesting subject of continued studies.

\section{EFFICACY OF RITUXIMAB IN TREATING PROTEINASE 3-ANCA VS. MYELOPEROXIDASE- ANCA}

During the RAVE trial, patient responses to RTX vs. CYC were analyzed based on ANCA-type (PR-3 vs. MPO). The data concluded that RTX was also superior to CYC in maintaining remission specifically in ANCA PR-3 positive patients $v s$. patients who were positive for ANCA-MPO ${ }^{[9]}$. The MAINRITSAN trial enrolled more patients who were positive for anti-PR-3 ANCA in the study than patients who were positive for anti-MPO ANCA, so the results were more indicative of the response of the anti-PR3 ANCA patient population to RTX [Table 1]. Earlier trials also enrolled greater numbers of PR-3 ANCA positive patients ${ }^{[17,21]}$. This poses an interesting question as to whether RTX is more effective in treating and preventing disease relapse in patients who are specifically PR-3-ANCA positive and, would be a good subject of future research.

\section{RISK FACTORS FOR DISEASE RELAPSE IN ANCA ASSOCIATED VASCULITIS}

The utility of monitoring the presence of autoreactive ANCA antibodies, ANCA titers and, presence of circulating $\mathrm{CD} 19+\mathrm{B}$ cells as a risk factor for disease relapse in AAV has been a matter of debate due to conflicting data on this subject throughout the years. It is particularly useful to know this information as prevention of relapses is paramount in reducing organ damage and increasing survival in AAV patients. There is conflicting evidence regarding this subject because while ANCA titers do often drastically decrease in patients who have received induction therapy, some patients who have achieved disease remission can remain ANCA positive and certain patients who are negative for ANCA autoantibodies can still relapse. The same question is posed regarding the presence of circulating CD19+ B cells as relapses have occurred regardless of the presence of $B$ cells in AAV patients. The MAINRITSAN 2 trial addresses this fact as the study notes that four patients experienced disease relapses despite being ANCA negative and having no B cells in the circulation. 
A single center cohort study conducted in June 2020 sought to investigate the role of the presence of ANCA autoantibodies, ANCA titers and, the appearance of CD19+ B cells in the circulation as predictors of the risk of disease relapse in AAV patients. The study found that ANCA negativity regardless of B cell presence was a strong predictor that disease remission would be maintained. Additionally, PR-3 ANCA positivity or persistence of PR-3 ANCA levels was a strong predictor of relapse. Relapses in the MPOANCA population occurred exclusively when $B$ cells were present. This research provides the interesting possibility that a tailored regimen of therapy based on ANCA positivity and presence of B cells could be effective in preventing disease relapse in AAV patients. The question that remains is if this method could lead to overtreatment, as some patients are able to achieve clinical remission despite ANCA positivity and relapses have been known to occur in patients regardless of $B$ cell presence. This will be an interesting topic of further research ${ }^{[40]}$.

\section{CONCLUSION}

Treatment of AAV has always provided a challenge for physicians both because of its severe, life threatening manifestations and, the multitude of side effects caused by the cytotoxic medications needed to induce and maintain disease remission. Despite these therapies, patients with AAV still experienced high rates of relapse. Cyclophosphamide was the gold standard of induction therapy for many years however, given its extensive risk of cytotoxic side effects, it was not an ideal long-term maintenance therapy for patients with AAV and other agents were explored for disease maintenance remission. Among these medications are MTX and AZA, which gained recognition for their ability to induce disease remission and shorten the length of time of CYC exposure, however, these medications had high relapse rates and were associated with numerous adverse effects. The RAVE and RITUXVAS trials demonstrated that RTX was non-inferior to CYC in successfully achieving disease remission in AAV patients and, had a similar safety profile to CYC.

After the discovery that RTX was a promising induction agent for AAV, early retrospective reviews were conducted exploring RTX as a maintenance therapy for AAV. The MAINRITSAN trial provided evidence of the superiority of RTX to AZA in preventing disease relapse in AAV patients, and the MAINRITSAN 2 trial demonstrated that individually tailored regimens of RTX infusions based on the reappearance of ANCA autoantibodies, increase of ANCA titers or, reappearance of CD19+ B lymphocytes from treatment randomization had similarly low relapse rates as fixed dose regimens of RTX and, enabled patients to receive fewer infusions. The RITAZAREM trial demonstrated the superiority of RTX as a maintenance therapy compared to AZA in the prevention of disease relapse in patients with a history of relapsing AAV and, showed that RTX had fewer associated adverse events. Additionally, despite being left out of the above trials due to its distinct pathogenesis, RTX maintenance was also investigated in EGPA as compared to CYC in another study and was deemed an effective and well tolerated treatment option for EGPA.

These studies are monumental in the advancement of therapy for AAV because up until recently, the gold standard induction therapy for this disease was extremely cytotoxic and even though alternate maintenance strategies were aimed at reducing the toxic effects of CYC, they had high rates of relapse and were associated with adverse effects of their own. The above literature reveals that RTX is a safe, effective and well tolerated induction and maintenance therapy in AAV that can be given in a tailored dosing regimen, thus exposing patients to fewer infusions. This has important implications in the treatment of AAV as patients treated with RTX will have better disease control with lower relapse rates, fewer exposures to the medication and, a decreased incidence of adverse events. As a result, patients with AAV receiving RTX therapy will have higher survival rates and longer relapse free periods, leading to an overall enhanced quality of life and improvement in patient outcomes. 


\section{DECLARATIONS}

\section{Authors' contributions}

Made substantial contributions to conception and design of this review and performed data interpretation: Skopis M, Bag-Ozbek A

\section{Availability of data and materials}

Not applicable.

\section{Financial support and sponsorship}

None.

\section{Conflicts of interest}

Both authors declared that there are no conflicts of interest.

\section{Ethical approval and consent to participate}

Not applicable.

\section{Consent for publication}

Not applicable.

\section{Copyright}

(c) The Author(s) 2020.

\section{REFERENCES}

1. Yates M, Watts R. ANCA-associated vasculitis. Clin Med (Lond) 2017;17:60-4.

2. Jennette JC, Nachman PH. ANCA Glomerulonephritis and Vasculitis. Clin J Am Soc Nephrol 2017;12:1680-91.

3. Fauci AS, Wolff SM. Wegner's Granulomatosis: studies in eighteen patients and a review of the literature. Medicine (Baltimore) 1973;52:535-61.

4. Hassan RI, Gaffo AL. Rituximab in ANCA-associated vasculitis. Curr Rheumatol Rep 2017;19:6.

5. de Groot K, Harper L, Jayne D, Felipe L, Gregorini G et al. Pulse versus oral cyclophosphamide for induction of remission in antineutrophil cytoplasmic antibody-associated vasculitis: a randomized trial. Ann Intern Med 2009;150:670-80.

6. De Groot K, Rasmussen N, Bacon PA, Tervaert JW, Feighery C, et al. Randomized trial of cyclophosphamide versus methotrexate for induction of remission in early systemic antineutrophil cytoplasmic antibody-associated vasculitis. Arthritis Rheum 2005;52:2461-9.

7. Jayne DR, Gaskin G, Rasmussen N, Abramowicz D, Ferrario F, et al; European Vasculitis Study Group. Randomized trial of plasma exchange or high-dosage methylprednisolone as adjunctive therapy for severe renal vasculitis. J Am Soc Nephrol 2007;18:2180-8.

8. Walsh M, Merkel PA, Peh CA, Szpirt WM, Puéchal X, et al; PEXIVAS Investigators. Plasma exchange and glucocorticoids in severe ANCA-associated vasculitis. N Engl J Med 2020;382:622-31.

9. Stone JH, Merkel PA, Spiera R, Seo P, Langford CA, et al; RAVE-ITN Research Group. Rituximab versus cyclophosphamide for ANCAassociated vasculitis. N Engl J Med 2010;363:221-32.

10. Jones RB, Tervaert JW, Hauser T, Luqmani R, Morgan MD, et al; European Vasculitis Study Group. Rituximab versus cyclophosphamide in ANCA-associated renal vasculitis. N Engl J Med 2010;363:211-20.

11. Jayne D, Rasmussen N, Andrassy K, Bacon P, Tervaert JW, et al; European Vasculitis Study Group. A randomized trial of maintenance therapy for vasculitis associated with antineutrophil cytoplasmic autoantibodies. N Engl J Med 2003;349:36-44.

12. Maritati F, Alberici F, Oliva E, Urban ML, Palmisano A, et al. Methotrexate versus cyclophosphamide for remission maintenance in ANCA-associated vasculitis: a randomised trial. PLoS One 2017;12:e185880.

13. Pagnoux C, Mahr A, Hamidou MA, Boffa JJ, Ruivard M, et al; French Vasculitis Study Group. Azathioprine or methotrexate maintenance for ANCA-associated vasculitis. N Engl J Med 2008;359:2790-803.

14. Randall KL. Rituximab in autoimmune diseases. Aust Prescr 2016;39:131-4.

15. Jennette JC, Falk RJ. B cell-mediated pathogenesis of ANCA-mediated vasculitis. Semin Immunopathol 2014;36:327-38.

16. Specks U, Fervenza FC, Mcdonald TJ, Hogan MCE. Response of Wegener's granulomatosis to anti-CD20 chimeric monoclonal antibody therapy. Arthritis Rheum 2001;44:2836-40.

17. Eriksson P. Nine patients with anti-neutrophil cytoplasmic antibody-positive vasculitis successfully treated with rituximab. J Intern Med 2005;257:540-8.

18. Bruhaye B, Aouba A, Pagnoux C, Lacassin F, Guillevin L. Adjunction of Rituximab to steroids and immunosuppressants for refractory/ 
relapsing Wegner's granulomatosis: a study on 8 patients. Clin Exp Rheumatol 2007;25:S23-7.

19. Jones RB, Ferraro AJ, Chaudhry AN, Brogan P, Salama AD, et al. A multicenter survey of rituximab therapy for refractory antineutrophil cytoplasmic antibody-associated vasculitis. Arthritis Rheum 2009;60:2156-68.

20. Rhee EP, Laliberte KA, Niles JL. Rituximab as maintenance therapy for anti-neutrophil cytoplasmic antibody-associated vasculitis. Clin J Am Soc Nephrol 2010;5:1394-400.

21. Cartin-Ceba R, Golbin JM, Keogh KA, Peikert T, Sánchez-Menéndez M, et al. Rituximab for remission induction and maintenance in refractory granulomatosis with polyangiitis (Wegener's): ten-year experience at a single center. Arthritis Rheum 2012;64:3770-8.

22. Baudron-Roubaud C, Pagnoux C, Ruault-Meaux N, Grasland A, Zoulim A, et al. Rituximab maintenance therapy for granulomatosis with polyangiitis and microscopic polyangiitis. $J$ Rheumatol 2012;39:125-30.

23. Guillevin L, Pagnoux C, Karras A, Khouatra C, Aumaître O, et al; French Vasculitis Study Group. Rituximab versus azathioprine for maintenance in ANCA-associated vasculitis. N Engl J Med 2014;371:1771-80.

24. Charles P, Terrier B, Perrodeau É, Cohen P, Faguer S, et al; French Vasculitis Study Group. Comparison of individually tailored versus fixed-schedule rituximab regimen to maintain ANCA-associated vasculitis remission: results of a multicentre, randomised controlled, phase III trial (MAINRITSAN2). Ann Rheum Dis 2018;77:1143-9.

25. Smith RM, Jayne D, Merkel P. A randomized, controlled trial of Rituximab versus Azathioprine after induction of remission with Rituximab for patients with ANCA-associated vasculitis and relapsing disease (RITAZAREM) [abstract]. Arthritis Rheumatol $2019 ; 71$. Available from: https:/acrabstracts.org/abstract/a-randomized-controlled-trial-of-rituximab-versus-azathioprine-after-induction-ofremission-with-rituximab-for-patients-with-anca-associated-vasculitis-and-relapsing-disease/. [Last accessed on 28 Sep 2020]

26. Henderson S, Copley S, Pusey C, Ind P, Salama A. Prolonged B cell depletion with Rituximab is effective in treating refractory pulmonary granulomatous inflammation in granulomatosis with polyangiitis (GPA). Medicine(Baltimore) 2014;93;e299.

27. Geetha D, Jefferson JA. ANCA-associated vasculitis: core curriculum 2020. Am J Kidney Dis 2020;75:124-37.

28. Geetha D, Hruskova Z, Segelmark M, Hogan J, Morgan M, et al. Rituximab for treatment of severe renal disease in ANCA associated vasculitis. J Nephrol 2016;29:195-201.

29. Recillas-Gispert C, Serna-Ojeda JC, Flores-Suárez LF. Rituximab in the treatment of refractory scleritis in patients with granulomatosis with polyangiitis (Wegener’s). Graefes Arch Clin Exp Ophthalmol 2015;253:2279-84.

30. Suhler EB, Lim LL, Beardsley RM, Giles TR, Pasadhika S, et al. Rituximab therapy for refractory orbital inflammation: results of a phase 1/2, dose-ranging, randomized clinical trial. JAMA Ophthalmol 2014;132:572-8.

31. Sharma A, Kumar S, Wanchu A, Lal V, Singh R, et al. Successful treatment of hypertrophic pachymeningitis in refractory Wegener's granulomatosis with rituximab. Clin Rheumatol 2010;29:107-10.

32. Lally L, Lebovics RS, Huang WT, Spiera RF. Effectiveness of rituximab for the otolaryngologic manifestations of granulomatosis with polyangiitis (Wegener's). Arthritis Care Res (Hoboken) 2014;66:1403-9.

33. Cartin-Ceba R, Diaz-Caballero L, Al-Qadi MO, Tryfon S, Fervenza FC, et al. Diffuse alveolar hemorrhage secondary to antineutrophil cytoplasmic antibody-associated vasculitis: predictors of respiratory failure and clinical outcomes. Arthritis Rheumatol 2016;68:1467-76.

34. Tieu J, Smith R, Basu N, Brogan P, D'Cruz D, et al. Rituximab for maintenance of remission in ANCA-associated vasculitis: expert consensus guidelines. Rheumatology (Oxford) 2020;59:e24-32.

35. Reitblat $\mathrm{T}$, Wechsler A, Reitblat O. Rituximab-related late-onset neutropenia in patients with rheumatic diseases: successful re-challenge of the treatment. Am J Case Rep 2015;16:211-4.

36. Berger JR, Malik V, Lacey S, Brunetta P, Lehane PB. Progressive multifocal leukoencephalopathy in rituximab-treated rheumatic diseases: a rare event. J Neurovirol 2018;24:323-31.

37. Segelmark L, Flores-suarez LF, Rathmann J, Mohammad A. 271. Severe infections in patients with anca-associated vasculitis treated with Rituximab. Rheumatology 2019;58:kez062.045.

38. Kronbichler A, Kerschbaum J, Gopaluni S, Tieu J, Alberici F, et al. Trimethoprim-sulfamethoxazole prophylaxis prevents severe/lifethreatening infections following rituximab in antineutrophil cytoplasm antibody-associated vasculitis. Ann Rheum Dis 2018;77:1440-7.

39. Thiel J, Troilo A, Salzer U, Schleyer T, Halmschlag K, et al. Rituximab as induction therapy in eosinophilic granulomatosis with polyangiitis refractory to conventional immunosuppressive treatment: a 36-month follow-up analysis. J Allergy Clin Immunol Pract 2017;5:1556-63.

40. van Dam LS, Dirikgil E, Bredewold EW, Ray A, Bakker JA, et al. Proteinase-3-anti-neutrophil cytoplasmic antibodies (PR3-ANCAs) predict relapses in ANCA-associated vasculitis patients after rituximab. Nephrol Dial Transplant 2020:gfaa066. 